\title{
Conservative arch management versus aggressive arch reconstruction for type $A$ intramural hematoma
}

\author{
Jin-Dong Li, Wei-Guo Ma, Li-Zhong Sun \\ Department of Cardiovascular Surgery, Beijing Anzhen Hospital, Capital Medical University, and Beijing Institute of Heart Lung and Blood Vessel \\ Diseases, Beijing 100029, China \\ Correspondence to: Li-Zhong Sun, MD. Department of Cardiovascular Surgery, Beijing Anzhen Hospital, Capital Medical University, Beijing 100029, \\ China. Email: lizhongsun@outlook.com.
}

Submitted Apr 02, 2019. Accepted for publication: Jun 28, 2019.

doi: 10.21037/acs.2019.07.02

View this article at: http://dx.doi.org/10.21037/acs.2019.07.02

The optimal management strategy of intramural hematomas (IMH) involving the ascending aorta (type A) remains controversial. While early operation is recommended in current western series (1-3), some Asian groups have obtained favorable clinical results with medical treatment (4-6). However, there still remains significant progression in a large proportion of patients undergoing late surgery despite optimized medical therapy, which suggests that an extensive approach to type A IMH may be warranted. The meta-analysis by Matsushita and colleagues (7) has illustrated that an aggressive surgical approach to type A IMH is safe and strongly recommended when there is evidence of cardiac tamponade, an ascending aortic diameter of $\geq 4.5 \mathrm{~cm}$, or a hematoma of $\geq 7 \mathrm{~mm}$ in thickness.

Surgical management of type A IMH remains a formidable challenge when the ascending aorta, arch, and part (or all) of the descending aorta are involved. Several surgical approaches have been employed, but the optimal technique is not clearly established and there is an ongoing controversy regarding the role of conservative arch management versus aggressive arch reconstruction in the management of type A IHM. In most series, conservative arch management with ascending aortic or a partial arch (hemiarch) replacement was performed in the majority of patients with type A IMH. For patients who are old, those with no hematoma in the arch and distal aorta, an arch diameter of $\leq 45 \mathrm{~mm}$ or no penetrating atherosclerotic ulcer (PAU) in the arch, the conservative approach may be a reasonable treatment option with certain advantages.

It is quicker by avoiding the need for deep hypothermic circulatory arrest and minimizes the risk of introducing air and debris into the arch, which may decrease the rates of operative mortality and morbidity. However, because IMH is a "radiographic diagnosis," the culprit lesion (intimal tear or ulceration) may not be identifiable during ascending or hemiarch repair for type A IMH, therefore it remains uncertain whether the initial culprit of IMH could be treated properly. Such patients remain at the risk of sustaining subsequent adverse events associated with unidentified lesions in other distal aortic segments. In their study, Matsushita and associates found $10 \%$ of patients underwent a reintervention at a median follow-up of 4 years; all these reoperations were performed on the distal aorta, with more than half involving the arch (7). With longer follow-up, it is likely that this number will increase.

This also raises the question of where a more aggressive strategy for arch repair fits into the treatment decision for type A IMHs, given the high incidence of reintervention and infrequent healing. In 66 patients followed for a mean of 7.6 years, $30.3 \%(20 / 66)$ had an increase in IMH size, development of an ulcer-like projection, or progression to a classic dissection and $48.5 \%$ (32/66) underwent surgery eventually (6). Sadamatsu and colleagues reported that late adverse events occurred in $15.8 \%$ of patients (3/19) at mean 1.6 years after surgery (4), including death, abdominal aortic aneurysm repair and middle colic artery aneurysm in one each.

These data suggest that a more aggressive arch reconstruction may be warranted. With clinical and radiologic evidence accrued in large series, total arch replacement (TAR) has been shown to be associated with favorable survival and freedom from reoperation for patients 

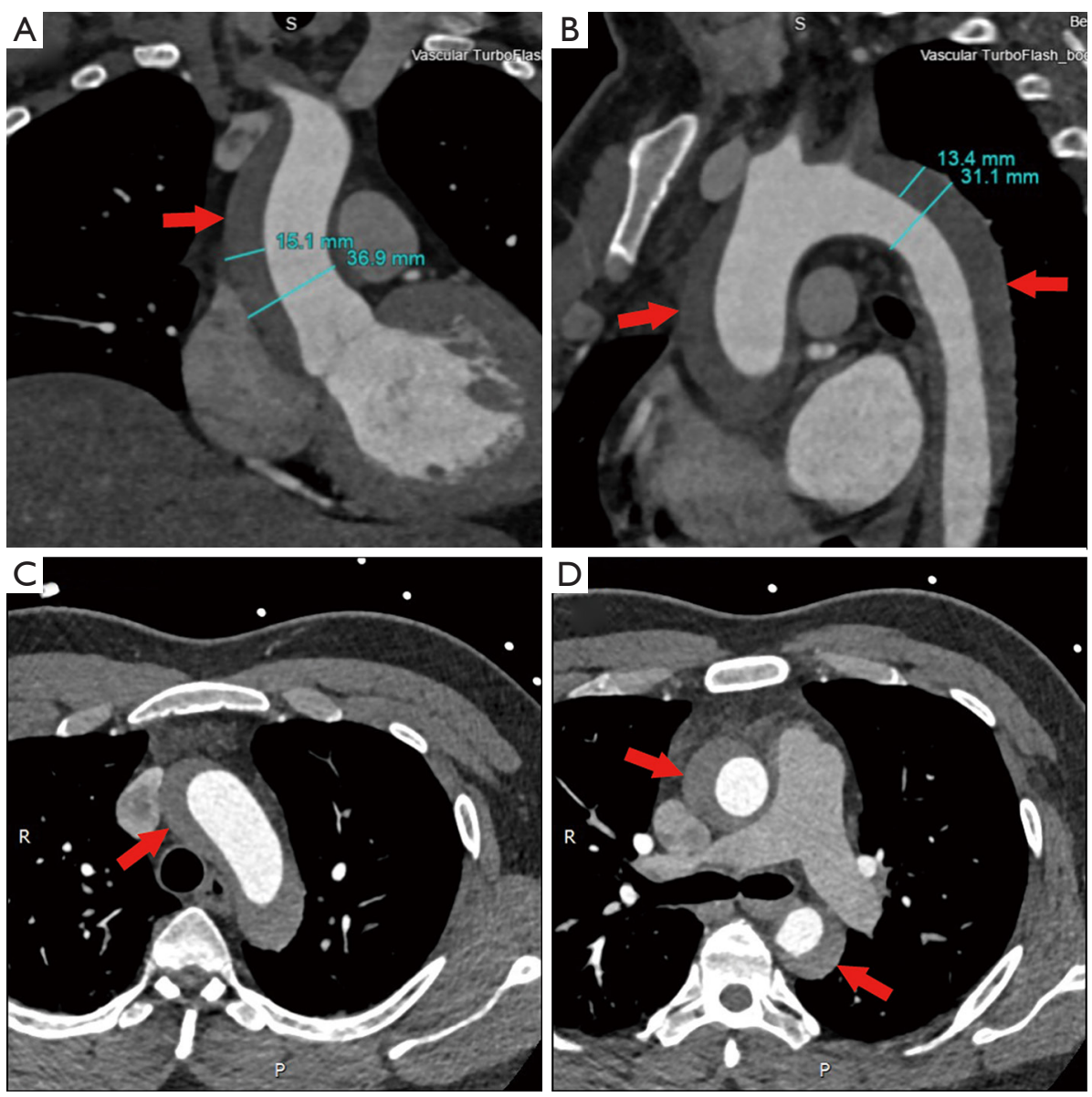

Figure 1 Computed tomographic angiography (CTA) prior to surgical repair reveals a type A intramural hematoma (IMH) involving the aortic arch in a 32-year-old male. (A) Coronal view shows the IMH involving the ascending aorta; (B) oblique sagittal view reveals the aortic arch and descending aorta were involved; (C) axial view demonstrates the IMH involves the aortic arch; (D) axial view reveals involvement of both the ascending and descending aorta. Note the typical crescentic shape of the IMH (arrows).

with acute type A dissection in the long term. However, this aggressive approach has not gained wide use in patients with acute type A IMH because of its inherent risks and technical complexity (8).

In our practice, we consider IMH as a precursor to classic dissection, depicted by radiological images at a specific phase in its clinical evolution. A lot of our patients with crescent IMH on preoperative CT scan (Figure 1) were found intraoperatively to have typical intimal tears with true and false lumens (Figure 2). Encouraged by our large experience and favorable surgical results, we routinely consider a more aggressive approach for type A IMH patients with the following conditions:

(I) An ascending or arch IMH of $\geq 10 \mathrm{~mm}$ in thickness;

(II) Dilatation of the ascending aorta $(\geq 5 \mathrm{~cm})$ or arch

\section{$(\geq 4.5 \mathrm{~cm}$ in diameter);}

(III) Concomitant PAU in the ascending aorta or the arch;

(IV) Concomitant intimal tear located in the distal arch or the greater curvature of the arch;

(V) Evidence of tamponade;

(VI) Uncontrollable chest or back pain.

In our team, we routinely perform TAR with frozen elephant trunk (FET) implantation (i.e., the Sun operation) in the majority of patients with type A IMH (9). This technique is particularly beneficial for patients with extensive IMH involving the aortic arch. In such patients, the intima of the aorta is extremely fragile, which makes it very difficult or impossible to place stitches securely for distal anastomosis during ascending aortic or hemiarch repair. In addition, suturing in the fragile arch may penetrate the IMH and the 

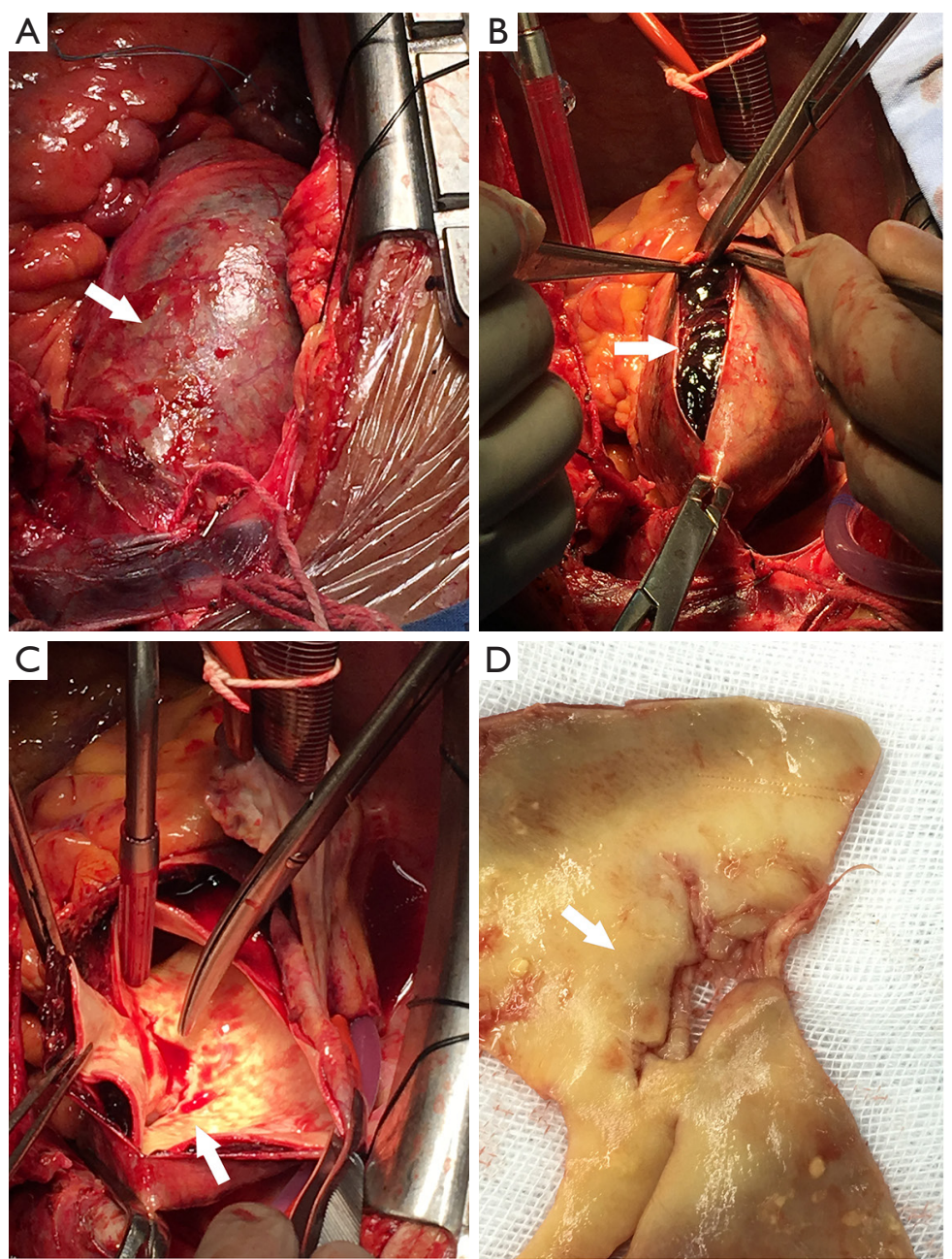

Figure 2 During surgery for the same patient, the IMH turned out to be a typical type A dissection. (A) The ascending aorta was enlarged, measuring $5.0 \mathrm{~cm}$ in diameter. Note the dark reddish color along its length (arrow); (B) thrombosed false lumen (arrow) was seen upon opening of the aortic adventitia; (C) intimal tear and flaps (arrow) were shown when the aortic wall was cut open; (D) resected aortic wall with an intimal tear (arrow), confirming a typical dissection. IMH, intramural hematoma.

false lumen, which runs very high risk of introducing thrombi into the aortic lumen, predisposing patients to cerebral thromboembolic complications. Our approach solves the above two issues simultaneously.

Excision of the diseased aortic segments and implantation of FET avoid the need for suturing of the brittle ascending aorta or arch, which minimizes the risk of anastomotic bleeding. At the same time, arch reconstruction with 4-branched graft eliminates the risk of thromboembolic events associated with hemiarch repair. An FET implanted in the descending aorta promotes absorption of the IMH and remodeling of the distal aorta, which can avoid or facilitate distal reintervention and improve survival in the long term (10).
Inspection of our own unpublished data proves the safety and efficacy of this aggressive approach. In 52 type A IMH patients (mean age $51.5 \pm 6.2$ years) undergoing TAR + FET, operative mortality and stroke occurred in one patient each (1.9\%); none sustained spinal cord injury; reoperation for bleeding and dialysis for acute kidney injury were required in $1(1.9 \%)$ and 2 each $(3.8 \%)$. Follow-up was available in $100 \%$ at mean $3.5 \pm 0.8$ years. One patient died of cerebral hemorrhage at 13 months. Disease progression occurred in 2 patients (3.9\%), including type B dissection at 3 months and distal stent-graft new entry at 1 year, in one each, which were successfully managed with endovascular repair. Two other endovascular reinterventions were performed for type 

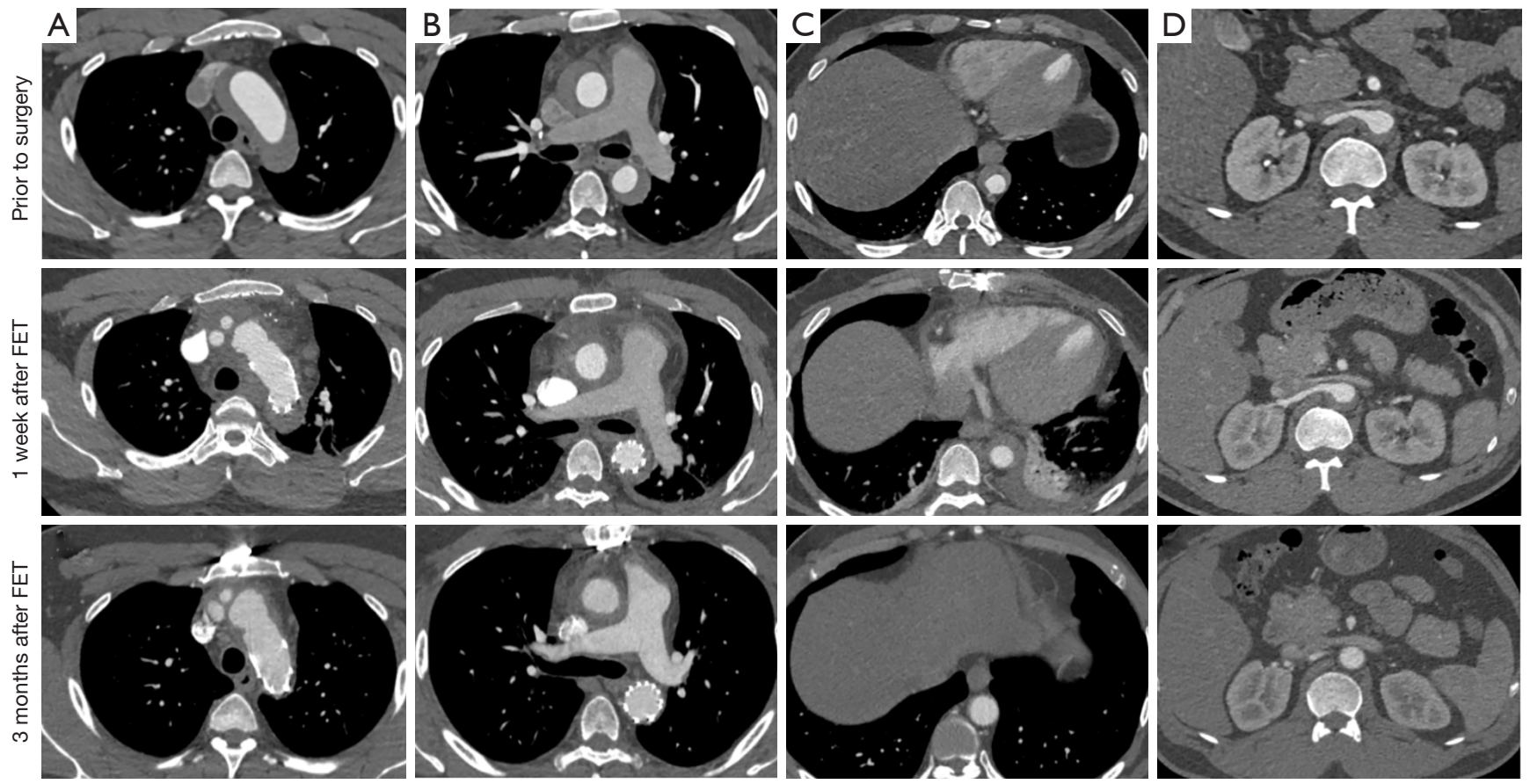

Figure 3 Serial CTA showing evolution of the type A IMH in the same patient. At the levels of the aortic arch (A), pulmonary artery bifurcation (B), diaphragmatic hiatus (C) and renal arteries (D), complete aortic remodeling was observed after the frozen elephant trunk procedure. At 3 months after surgery, CTA demonstrated false lumen obliteration and absorption of the IMH in this patient. CTA, computed tomographic angiography; IMH, intramural hematoma.

Ia endoleak at 13 months and ascending aortic anastomotic leak at 10 months, in one patient each. The rates of distal hematoma absorption were $68.6 \%, 84.7 \%$ and $94.8 \%$ at 1 , 3 and 6 months after surgery, respectively (Figure 3).

In conclusion, a more aggressive surgical strategy using the TAR and FET technique is justified in selected patients with type A IMH, which should be tailored individually to pathologic features, patient condition and surgeon experience.

\section{Acknowledgments}

Funding: This work was supported in part by the Beijing Major Science and Technology Projects from Beijing Municipal Science and Technology Commission (No. Z171100001017083).

\section{Footnote}

Conflicts of Interest: The authors have no conflicts of interest to declare.

\section{References}

1. Evangelista A, Mukherjee D, Mehta RH, et al. Acute intramural hematoma of the aorta: a mystery in evolution. Circulation 2005;111:1063-70.

2. Estrera AL, Sandhu HK, Leake SS, et al. Early and late outcomes of acute type A aortic dissection with intramural hematoma. J Thorac Cardiovasc Surg 2015;149:137-42.

3. Chou AS, Ziganshin BA, Charilaou P, et al. Long-term behavior of aortic intramural hematomas and penetrating ulcers. J Thorac Cardiovasc Surg 2016;151:361-72, 373.e1.

4. Sadamatsu K, Takase S, Sagara S, et al. Initial medical management in acute type A aortic dissection patients with a thrombosed false lumen in the ascending aorta combining intramural hematoma and retrograde dissection from the descending to the ascending aorta. Eur Heart J Acute Cardiovasc Care 2018:2048872618777724. [Epub ahead of print].

5. Watanabe $S$, Hanyu $M$, Arai $Y$, et al. Initial medical treatment for acute type A intramural hematoma and 
aortic dissection. Ann Thorac Surg 2013;96:2142-6.

6. Kitai T, Kaji S, Yamamuro A, et al. Clinical outcomes of medical therapy and timely operation in initially diagnosed type A aortic intramural hematoma: a 20-year experience. Circulation 2009;120:S292-8.

7. Matsushita A, Fukui T, Tabata M, et al. Preoperative characteristics and surgical outcomes of acute intramural hematoma involving the ascending aorta: A propensity score-matched analysis. J Thorac Cardiovasc Surg 2016;151:351-8.

8. Shimokawa T, Ozawa N, Takanashi S, et al. Intermediate- term results of surgical treatment of acute intramural hematoma involving the ascending aorta. Ann Thorac Surg 2008;85:982-6.

9. Ma WG, Zheng J, Liu YM, et al. Dr. Sun's procedure for type A aortic dissection: Total arch replacement using tetrafurcate graft with stented elephant trunk implantation. Aorta (Stamford) 2013;1:59-64.

10. Chen Y, Ma WG, Zhi AH, et al. Fate of distal aorta after frozen elephant trunk and total arch replacement for type A aortic dissection in Marfan syndrome. J Thorac Cardiovasc Surg 2018;157:835-49.
Cite this article as: Li JD, Ma WG, Sun LZ. Conservative arch management versus aggressive arch reconstruction for type A intramural hematoma. Ann Cardiothorac Surg 2019;8(5):551555. doi: 10.21037/acs.2019.07.02 